\title{
Sub-40-fs high-power Yb:CALYO laser pumped by single-mode fiber laser
}

\author{
Wenlong Tian ${ }^{\oplus 1}$, Geyang Wang ${ }^{1}$, Dacheng Zhang ${ }^{1}$, Jiangfeng Zhu ${ }^{1}$, Zhaohua Wang ${ }^{2}$, Xiaodong $\mathrm{Xu}^{3}$, \\ Jun $\mathrm{Xu}^{4}$, and Zhiyi $\mathrm{Wei}^{2}$ \\ ${ }^{1}$ School of Physics and Optoelectronic Engineering, Xidian University, Xi'an 710071, China \\ ${ }^{2}$ Beijing National Laboratory for Condensed Matter Physics, Institute of Physics, Chinese Academy of Sciences, Beijing 100190, China \\ ${ }^{3}$ Jiangsu Key Laboratory of Advanced Laser Materials and Devices, School of Physics and Electronic Engineering, Jiangsu Normal \\ University, Xиzhou 221116, China \\ ${ }^{4}$ School of Physics Science and Engineering, Tongji University, Shanghai 200092, China \\ (Received 14 July 2019; revised 15 October 2019; accepted 7 November 2019)
}

\begin{abstract}
We report on the study of single-mode fiber-laser-pumped mode-locked Yb:CALYO lasers via using a passive saturable absorber and Kerr-lens mode-locking technique, respectively. Up to 3.1-W average power with 103-fs pulse duration is obtained from the passive mode-locking, and down to 36-fs pulse duration with more than 2-W average power is achieved by the pure Kerr-lens mode-locking, which is to the best of our knowledge, the highest average power from a reported sub-40-fs Yb-based solid-state oscillator.
\end{abstract}

Keywords: fiber pump; high power; solid-state laser; sub-40-fs; Yb:CALYO

\section{Introduction}

All-solid-state femtosecond lasers that deliver high peak power and extremely narrow pulse width are of great interest in many fields such as scientific research, industry, medical treatment, and high speed communication. In particular, ytterbium-based solid-state lasers have attracted increasing attention because of their excellent optical and thermal properties. Recently, a great number of Yb-doped materi-

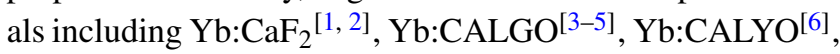

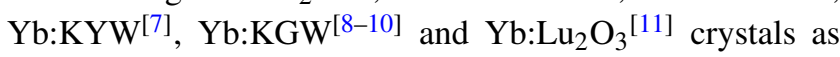
well as $\mathrm{Yb}: \mathrm{LuAG}$ ceramic $^{[12]}$ have shown their capacity in generating sub-100-fs pulses with watt level average power via either pure Kerr-lens mode-locking (KLM) or combination of KLM and saturable absorber-based passive mode-locking. Among them, Yb-doped calcium aluminate crystals including $\mathrm{Yb}: \mathrm{CALGO}$ and $\mathrm{Yb}: \mathrm{CALYO}$ are the most attractive candidates for high-power sub-50-fs pulses generation, due to their flat emission spectra with more than $70 \mathrm{~nm}$ bandwidths as well as high thermal conductivity. Regarding sub-50-fs pulses generation, Yb:CALGO and Yb:CALYO crystals have already been proven their abilities

Correspondence to: J. Zhu, No. 2 South Taibai Road, Xi'an 710071, China, Email: jfzhu@xidian.edu.cn; Z. Wei, Institute of Physics, Chinese Academy of Sciences, P.O. Box 603, Beijing 100190, China, Email: zywei@iphy.ac.cn by Zaouter et al. ${ }^{[13]}$, Agnesi et al. ${ }^{[14]}$, Gao et al. ${ }^{[15]}$ and Ma et al. ${ }^{[16]}$, respectively. Up to more than $3 \mathrm{~W}$ average power with 45 -fs pulses was obtained by Manjooran et al. ${ }^{[5]}$. Still, most of the obtained average powers from above literatures were only on the order of several tens of milliwatt, due to either the small pump power of the single-mode fiber coupled laser diodes (LDs) or the critical cavity setup restrained by the low-brightness multi-mode LDs. One way to cope with the issue raised by the low-brightness LDs is to use a double confocal cavity which separates the gain medium and Kerr medium. Paradis et al. demonstrated the generations of $35-\mathrm{fs}$ pulses with $1.5-\mathrm{W}$ average power and 49-fs pulses with 4.5-W average power from the KLM $\mathrm{Yb}: \mathrm{Lu}_{2} \mathrm{O}_{3}$ thin-disk laser, respectively, with $76-\mathrm{W}$ and $96-$ $\mathrm{W}$ pump power, ${ }^{[17]}$. However, the corresponding opticalto-optical efficiency is only $2.1 \%$ and $4.7 \%$, respectively. In terms of the KLM Yb:CALGO thin-disk laser, although down to 30 -fs pulses were delivered, the average power was only $150 \mathrm{~mW}$, due to the low gain and limited disk quality ${ }^{[18]}$. And for Yb:CALYO thin-disk lasers, no relevant mode-locking result has been reported so far. On the other hand, compared to the low-power single-mode fiber coupled LDs, single-mode fiber laser emitting at $976 \mathrm{~nm}$ emerging recently has not only higher average power up to $10 \mathrm{~W}^{[19]}$, but also excellent beam quality with $M^{2}<1.1$, distinct linear 
polarization characteristic as well as much lower noise. As a result, it is of great interest to generate sub-50-fs pulses with high average power by pumping the Yb:CALGO or Yb:CALYO crystal with a single-mode fiber laser. In 2014, Sévillano et al. demonstrated a single-mode fiber-laserpumped pure KLM Yb:CALGO oscillator, delivering 40-fs pulses with $1.1-\mathrm{W}$ average power ${ }^{[3]}$. However, preliminary results of only $250-\mathrm{mW}$ output power and 57 -fs pulse duration were generated from a single-mode fiber-laser-pumped $\mathrm{Yb}$ :CALYO laser ${ }^{[20]}$, which is far away from the expectation of generating much higher output power with shorter pulse duration considering the good optical and thermal properties of the Yb:CALYO crystal.

In this letter, we demonstrated the single-mode fiberlaser-pumped high-power Yb:CALYO lasers passively mode-locked by semiconductor saturable absorption mirror (SESAM) and pure Kerr-lens mode-locking, respectively. 103-fs pulses with up to 3.1-W average power are obtained from passive mode-locking, corresponding to the opticalto-optical efficiency of $31 \%$. In addition, as short as 36-fs pulses with up to $2-\mathrm{W}$ average power are obtained from the pure KLM with extra-cavity dispersion compensation. With the repetition rate of $84 \mathrm{MHz}$, the pulse energies and peak powers for SESAM-based passive mode-locking and KLM are $37 \mathrm{~nJ}, 0.31 \mathrm{MW}$ and $24 \mathrm{~nJ}, 0.58 \mathrm{MW}$, respectively.

\section{Experimental setup}

A schematic diagram of the experimental setup is shown in Figure 1. The pump source is a commercial single-mode fiber laser emitting at $976 \mathrm{~nm}$ with up to $10-\mathrm{W}$ output power (ALS-IR-75, Azur Light Systems). The pump beam was focused by a lens of 60- $\mathrm{mm}$ focal length into a 6-mm long Yb:CALYO crystal with a waist of $26.5 \mu \mathrm{m}\left(1 / e^{2}\right)$ and a Rayleigh length of $2.26 \mathrm{~mm}$. The 5 at. $\% \mathrm{Yb}^{3+}$-doped gain medium with anti-reflection coating around $980-1100 \mathrm{~nm}$ on both surfaces was wrapped with an indium foil and tightly mounted in a water-cooled copper heat sink to maintain at $14^{\circ} \mathrm{C}$ during the experiment. A half-wave plate was used to adjust the polarization of the pump beam since the absorption of Yb:CALYO crystal for $\pi$ polarization is much higher than that for $\sigma$ polarization. $\mathrm{C} 1$ and $\mathrm{C} 2$ were two dichroic concave mirrors with $75-\mathrm{mm}$ radius of curvature (ROC) and coated with high reflectivity over 1020-1100 $\mathrm{nm}(R>99.9 \%)$ and high transmittance around $800-1000 \mathrm{~nm}(R<5 \%)$. Several Gires-Tournois interferometer (GTI) mirrors were employed in the cavity for intra-cavity dispersion compensation. C3 was a concave mirror with ROC of $300 \mathrm{~mm}$, coated with high reflectivity in the region of $1020-1100 \mathrm{~nm}(R>99.9 \%)$. Wedged output couplers (OCs) were used to couple out the laser while avoiding mode-locking instability from the back reflection. The total cavity length was about $1.8 \mathrm{~m}$, corresponding to $84 \mathrm{MHz}$ repetition rate.

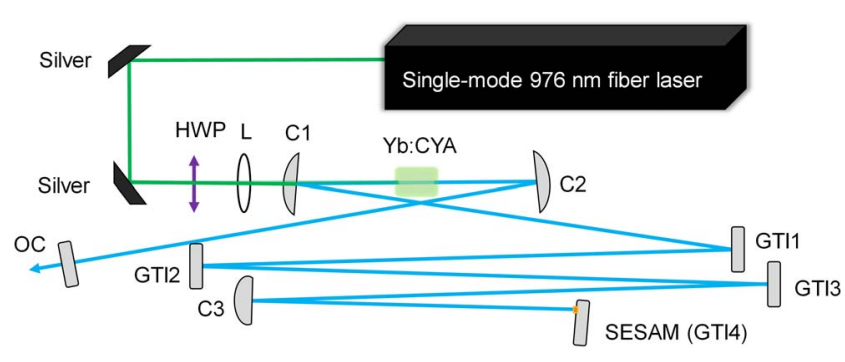

Figure 1. The sketch for the experimental setup of the single-mode fiberlaser-pumped mode-locked Yb:CALYO laser. HWP: half-wave plate; L: lens; C1-C3: concave mirrors; GTI1-GTI4: Gires-Tournois interferometer mirrors; OC: output coupler; SESAM: semiconductor saturable absorption mirror.

\section{Results and discussion}

At first, the cavity was optimized in a continuous-wave (CW) operation, which delivers 4.2 or $3.6-\mathrm{W}$ output powers with $15 \%$ or $10 \%$ OCs. The absorption efficiency of the crystal for pump laser is up to $99.5 \%$ under lasing. Then, to investigate the performance of a passive modelocking operation, a commercial SESAM (SAM-1040-0.71ps-x from BATOP GmbH) with $0.4 \%$ modulation depth at $1040 \mathrm{~nm}$ was utilized. With $10 \%$ transmittance OC, a stable mode-locking operation with up to 3.1-W average power was achieved when the total group delay dispersion (GDD) per round trip introduced by the three GTIs was $-6600 \mathrm{fs}^{2}$ (the GDD of GTI1, GTI2 and GTI3 per bounce is $-1000 \mathrm{fs}^{2}$, $-1000 \mathrm{fs}^{2}$ and $-1300 \mathrm{fs}^{2}$, respectively). The corresponding optical-to-optical efficiency is more than $30 \%$. Operating in open air, the root mean square of power fluctuation over half an hour was around $1.8 \%$. We characterized the optical spectrum as well as the temporal property via a commercial optical spectrum analyzer (AvaSpec-ULS2048, Avantes) and an intensity auto-correlator (APE PulseCheck USB), respectively. As shown in Figure 2(a), the optical spectrum of the pulses was centered at $1060.6 \mathrm{~nm}$, with the full-width at half-maximum (FWHM) bandwidth of $11.7 \mathrm{~nm}$. The corresponding intensity auto-correlation trace has an FWHM duration of 159 fs, as shown in Figure 2(b). If assuming a sech ${ }^{2}$ pulse shape, it results in a pulse duration of $103 \mathrm{fs}$. The corresponding time-bandwidth product was 0.327 , which is very close to the transform-limited value of 0.315 . Provided the $84 \mathrm{MHz}$ repetition rate, the corresponding single pulse energy and peak power were $37 \mathrm{~nJ}$ and $0.31 \mathrm{MW}$, respectively.

For pure Kerr-lens mode-locking operation, the SESAM was replaced by a fourth GTI mirror. When the dispersion provided by GTI4 per bounce was $-1000 \mathrm{fs}^{2}$, several different KLM operations were able to be realized by tuning the position of GTI4 close to the stability edge of the cavity. Because of the excellent beam quality of the pump laser and proper cavity design, soft-aperture type of KLM is easy to realize. When just slightly detuning the cavity with a distance of $\Delta L=-0.660 \mathrm{~mm}$ (the position of 

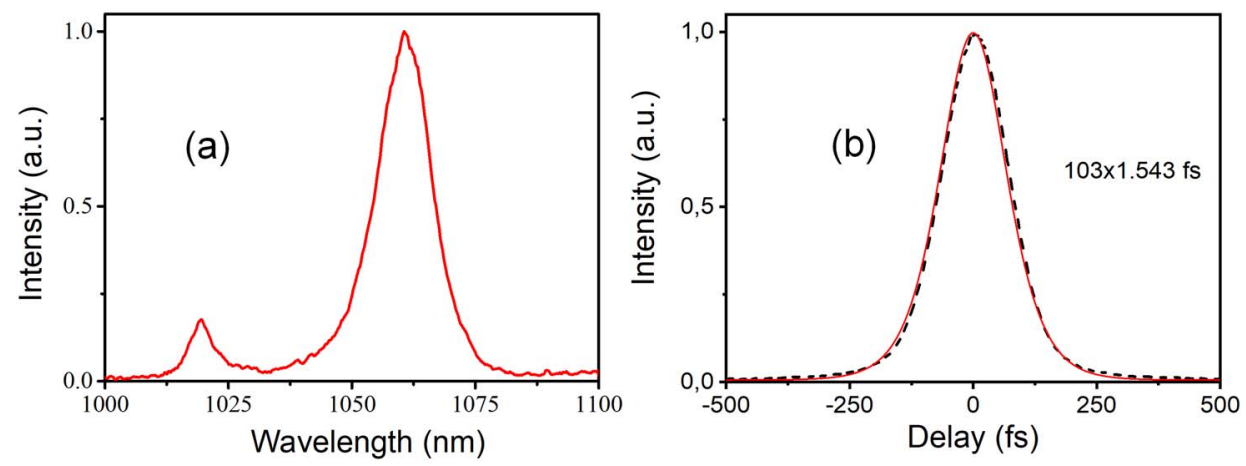

Figure 2. (a) Optical spectrum and (b) the corresponding auto-correlation trace of the passively mode-locked Yb:CALYO laser. The black dot and red solid curve in (b) stand for the experimental data and $\operatorname{sech}^{2}$-fitting, respectively.
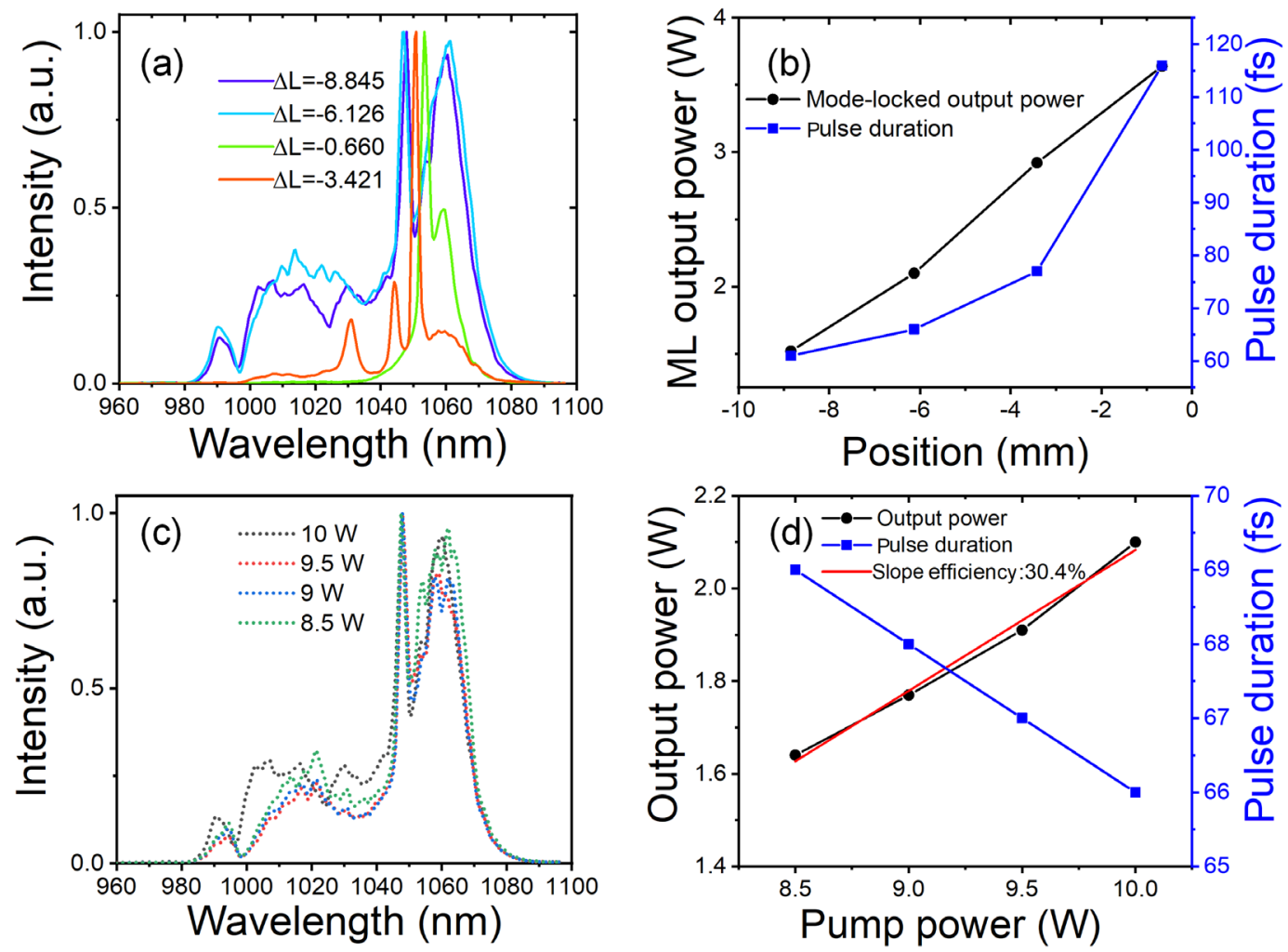

Figure 3. (a) Spectra; (b) output powers and pulse durations of different pure KLM operations realized by detuning the cavity with $\Delta L$; output properties under different pump power for KLM operation at $\Delta L=-6.126 \mathrm{~mm}$ : (c) evolution of spectra and (d) changing in average power as well as pulse duration.

$\Delta L=0 \mathrm{~mm}$ is where the maximum $\mathrm{CW}$ output power is produced), initial KLM operation would be obtained by a weak perturbation such as taping the mirrors or pushing the translation stage. The corresponding average power and pulse duration were $3.64 \mathrm{~W}$ and $116 \mathrm{fs}$, respectively. Then by further reducing the distance between C3 and GTI4, shorter pulses with broader spectrum were produced at the cost of linear decrease of the output power. The closer the cavity is to the edge of a stable region, the deeper the modulation depth of KLM is, resulting in higher loss. As shown in Figures 3(a) and 3(b), we recorded and compared the performances of different KLM operations versus the position of GTI4 mirror. The broadest spectrum was obtained at $\Delta L=-6.126 \mathrm{~mm}$, which covers from 980 to $1090 \mathrm{~nm}$. The lower intensity at shorter wavelength was due to the relatively high re-absorption of Yb:CALYO crystal. Via Fourier transform with zero chirps, the transform-limited pulse duration is $34 \mathrm{fs}$, meaning that there is residual chirp in the directly measured 66-fs pulses. At the position of $\Delta L=-8.845 \mathrm{~mm}$, the spectrum did not get broadened while the pulse duration was shortened to $61 \mathrm{fs}$, where the average power remained $1.52 \mathrm{~W}$. Due to the decreasing of average power, it was not able to support the stable KLM operation when the absolute value of $\Delta L$ was over $8.9 \mathrm{~mm}$. 

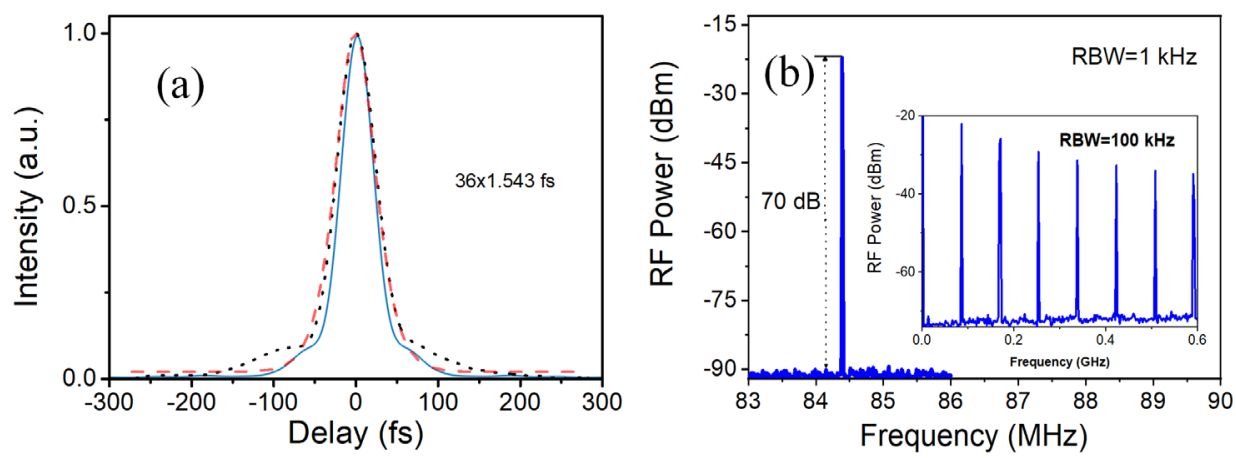

Figure 4. (a) The auto-correlation trace of the compressed pulses. Black and red curves are experimental result and sech ${ }^{2}$ fitting, respectively. The blue curve is the auto-correlation trace of the Fourier transformation of the corresponding optical spectrum. (b) The corresponding RF spectrum of the 36-fs pulses measured with frequency windows of $3 \mathrm{MHz}$ at $1 \mathrm{kHz}$ of RBW and $0.6 \mathrm{GHz}$ at $100 \mathrm{kHz}$ of RBW (inset), respectively.

For a given position of GTI4 $(\Delta L=-6.126 \mathrm{~mm})$, we characterized the performances of KLM pulses at different pump powers, as shown in Figures 3(c) and 3(d). It is clear that the pulse duration and average power of the laser pulses were decreasing, and linearly increasing with the rising of pump power, while their spectra did not change a lot. The corresponding slope efficiency of such a KLM operation was $30.4 \%$. At the maximum pump power of $10 \mathrm{~W}$, neither obvious thermal distortion nor multi-pulse operation was observed, indicating that the maximum output power of $2.1 \mathrm{~W}$ was limited by the limited pump power, and higher output power is possible with higher pump power.

To reduce the residual chirp of the femtosecond pulses, extra-cavity compression was implemented by using a pair of GTI mirrors with $-950 \mathrm{fs}^{2}$ GDD in total. As a result, down to $36-\mathrm{fs}$ pulses were obtained assuming a sech ${ }^{2}$ shape; the corresponding auto-correlation trace is shown in Figure 4(a). Meanwhile, the average power remained $2 \mathrm{~W}$, which is believed to be the highest average power from the reported sub-40-fs Yb-based solid-state oscillators. The corresponding single pulse energy and peak power were $24 \mathrm{~nJ}$ and $0.58 \mathrm{MW}$, respectively.

To claim the status of the KLM operation, the radio frequency (RF) spectra were measured via a photodetector (PD) with bandwidth of $1 \mathrm{GHz}$ and a commercial RF spectrum analyzer (Agilent E4407B). As described in Figure 4(b) is the RF spectrum of the compressed pulses of KLM operation at $\Delta L=-6.126 \mathrm{~mm}$. The signal was recorded for frequency spans of both $3 \mathrm{MHz}$ with $1 \mathrm{kHz}$ resolution bandwidth (RBW) and $0.6 \mathrm{GHz}$ with $100 \mathrm{kHz}$ RBW. The distinct signal-to-noise ratio of the fundamental beat note at $84.3 \mathrm{MHz}$ was as high as $70 \mathrm{~dB}$. No obvious side peaks of the several harmonics of the fundamental frequency were observed, which indicates that the KLM operation was running stably. Due to the excellent beam quality of the single-mode fiber laser as well as the well-designed cavity, the pure KLM operation could last for several tens of hours in open air once started.
Table 1. Overview of the state-of-the-art performance of ultrafast Yb solid-state oscillators generating sub-40-fs pulses. ${ }^{\text {a }}$

\begin{tabular}{|c|c|c|c|c|c|}
\hline Crystal & Geometry & $\bar{\tau}$ & $P_{\text {out }}$ & Energy & Reference \\
\hline Yb:CALGO & Bulk & $40 \mathrm{fs}$ & $15 \mathrm{~mW}$ & - & [14] \\
\hline Yb:CALGO & Bulk & $37 \mathrm{fs}$ & $1.5 \mathrm{~W}$ & $\sim 16 \mathrm{~nJ}$ & [3] \\
\hline Yb:CALGO & Bulk & $32 \mathrm{fs}$ & $90 \mathrm{~mW}$ & $\sim 1 \mathrm{~nJ}$ & [3] \\
\hline Yb:CALGO & Bulk & $39 \mathrm{fs}$ & $800 \mathrm{~mW}$ & $19 \mathrm{~nJ}$ & [5] \\
\hline Yb:CALGO & TDL & $30 \mathrm{fs}$ & $150 \mathrm{~mW}$ & $1.2 \mathrm{~nJ}$ & [18] \\
\hline Yb:CALYO & Bulk & $33 \mathrm{fs}$ & $36 \mathrm{~mW}$ & $0.3 \mathrm{~nJ}$ & [15] \\
\hline Yb:CALYO & Bulk & $30 \mathrm{fs}$ & $26 \mathrm{~mW}$ & $\sim 0.2 \mathrm{~nJ}$ & [16] \\
\hline Yb:YAG & Bulk & $35 \mathrm{fs}$ & $107 \mathrm{~mW}$ & $\sim 0.8 \mathrm{~nJ}$ & [21] \\
\hline Yb:YCOB & Bulk & $35 \mathrm{fs}$ & $36 \mathrm{~mW}$ & $\sim 0.4 \mathrm{~nJ}$ & [22] \\
\hline $\mathrm{Yb}: \mathrm{Lu}_{2} \mathrm{O}_{3}$ & TDL & $35 \mathrm{fs}$ & $1.6 \mathrm{~W}$ & $30 \mathrm{~nJ}$ & [17] \\
\hline Yb:CALYO & Bulk & $36 \mathrm{fs}$ & $2 \mathrm{~W}$ & $24 \mathrm{~nJ}$ & This work \\
\hline
\end{tabular}

${ }^{\text {a }} \tau$, pulse duration; $P_{\text {out }}$, average output power; TDL, thin-disk laser.

\section{Conclusion}

In conclusion, regarding the excellent optical property of the Yb:CALYO crystal, we have studied the KLM Yb:CALYO laser pumped by a single-mode fiber laser. Using an output coupler of $15 \%$ transmittance, different states have been realized by detuning the cavity. As the cavity was getting closer to the edge of stable region, the Kerr strength increased so that the modulation depth was rising accordingly. As a result, broader spectra as well as shorter pulses were obtained. The broadest spectrum was able to cover the wavelength from 980 to $1090 \mathrm{~nm}$, which supports 34-fs pulses with zero chirp. By extra-cavity chirp compensation, near transform-limited pulses of $36 \mathrm{fs}$ have been demonstrated. The average power after compression was $2 \mathrm{~W}$, meaning that the single pulse energy and peak power were up to $24 \mathrm{~nJ}$ and $0.67 \mathrm{MW}$, respectively. An overview of the state-of-the-art performance of ultrafast $\mathrm{Yb}$ solid-state oscillators generating sub-40-fs pulses is given in Table 1 .

Compared to the previous works in sub-40-fs generation from $\mathrm{Yb}$ solid-state lasers, we have presented, to the best of our knowledge, the highest average power as well as the largest single pulse energy within bulk geometry. Since no 
saturation in average power and multi-pulse operation was observed, sub-50-fs pulses with higher average power are believed to produce from such a single-mode fiber-laserpumped Yb:CALYO laser with higher pump power or longer crystal. It is noticed that the shorter wavelength $(980 \mathrm{~nm}-$ $1040 \mathrm{~nm}$ ) of the spectrum has relatively lower intensity due to the re-absorption. Another possibility is using a gainmatched output coupler ${ }^{[23]}$ to realize smoother spectrum, which is possible to support pulses with only a few cycles of pulse duration. Above all, the broad spectrum as well as good power stability of the laser provides a unique ultrafast source for seeding the femtosecond solid-state amplifier.

\section{Acknowledgements}

This research has been supported by the National Key Basic Research Program of China (No. 2017YFB0405202), National Natural Science Foundation of China (NSFC) (Nos. 61705174 and 11774277), Natural Science Basic Research Plan in Shaanxi Province of China (No. 2018JQ6061) and Open Research Fund of the State Key Laboratory of Pulsed Power Laser Technology, Electronic Engineering Institute, Hefei, China (No. SKL2017KF04).

\section{References}

1. F. Pirzio, S. D. Di Dio Cafiso, M. Kemnitzer, F. Kienle, A. Guandalini, J. Aus der Au, and A. Agnesi, J. Opt. Soc. Am. B 32, 2321 (2015).

2. G. Machinet, P. Sevillano, F. Guichard, R. Dubrasquet, P. Camy, J. L. Doualan, R. Moncorgé, P. Georges, F. Druon, D. Descamps, and E. Cormier, Opt. Lett. 38, 4008 (2013).

3. P. Sévillano, P. Georges, F. Druon, D. Descamps, and E. Cormier, Opt. Lett. 39, 6001 (2014).

4. A. Greborio, A. Guandalini, and J. Aus der Au, Proc. SPIE 8235, 823511 (2012).
5. S. Manjooran and A. Major, Opt. Lett. 43, 2324 (2018).

6. W. Tian, Y. Peng, Z. Zhang, Z. Yu, J. Zhu, X. Xu, and Z. Wei, Photon. Res. 6, 127 (2018).

7. F. M. Bain, A. A. Lagatsky, C. T. A. Brown, and W. Sibbett, Proc. SPIE 6871, 68712L (2008).

8. H. Zhao and A. Major, Opt. Express 21, 31846 (2013).

9. V. E. Kisel, A. S. Rudenkov, A. A. Pavlyuk, A. A. Kovalyov, V. V. Preobrazhenskii, M. A. Putyato, N. N. Rubtsova, B. R. Semyagin, and N. V. Kuleshov, Opt. Lett. 40, 2707 (2015).

10. R. Akbari and A. Major, Appl. Opt. 56, 8838 (2017).

11. M. Tokurakawa, A. Shirakawa, K. Ueda, R. Peters, S. T. Fredrich-Thornton, K. Petermann, and G. Huber, Opt. Express 19, 2904 (2011).

12. S. Kitajima, H. Nakao, A. Shirakawa, H. Yagi, and T. Yanagitani, Opt. Lett. 41, 4570 (2016).

13. Y. Zaouter, J. Didierjean, F. Balembois, G. Lucas Leclin, F. Druon, P. Georges, J. Petit, P. Goldner, and B. Viana, Opt. Lett. 31, 119 (2006).

14. A. Agnesi, Al. Greborio, F. Pirzio, G. Reali, J. Aus der Au, and A. Guandalini, Opt. Express 20, 10077 (2012).

15. Z. Gao, J. Zhu, J. Wang, Z. Wei, X. Xu, L. Zheng, L. Su, and J. Xu, Photon. Res. 3, 335 (2015).

16. J. Ma, H. Huang, K. Ning, X. Xu, G. Xie, L. Qian, K. P. Loh, and D. Tang, Opt. Lett. 41, 890 (2016).

17. C. Paradis, N. Modsching, V. J. Wittwer, B. Deppe, C. Kränkel, and T. Südmeyer, Opt. Express 25, 14918 (2017).

18. N. Modsching, C. Paradis, F. Labaye, M. Gaponenko, I. J. Graumann, A. Diebold, F. Emaury, V. J. Wittwer, and T. Südmeyer, Opt. Lett. 43, 879 (2018).

19. J. Wu, X. Zhu, H. Wei, K. Wiersma, M. Li, J. Zong, A. Chavez-Pirson, V. Temyanko, L. J. LaComb, R. A. Norwood, and N. Peyghambarian, Opt. Lett. 43, 951 (2018).

20. Z. Yu, H. Han, Y. Xie, Y. Peng, X. Xu, and Z. Wei, Opt. Express 24, 3103 (2016).

21. S. Uemura and K. Torizuka, Jpn. J. Appl. Phys. 50, 010201 (2011).

22. A. Yoshida, A. Schmidt, V. Petrov, C. Fiebig, G. Erbert, J. Liu, H. Zhang, J. Wang, and U. Griebner, Opt. Lett. 36, 4425 (2011).

23. C. Cihan, E. Beyatli, F. Canbaz, L. Chen, B. Sumpf, G. Erbert, A. Leitenstorfer, F. X. Kartner, A. Sennaroglu, and U. Demirbas, IEEE J. Sel. Top. Quantum Electron. 21, 94 (2015). 